TABLE

\begin{tabular}{cc}
\hline $\mathbf{X}$ & $\boldsymbol{P}$ \\
\hline 0 & 0.0115 \\
1 & 0.0577 \\
2 & 0.1369 \\
3 & 0.2053 \\
4 & 0.2182 \\
5 & 0.1746 \\
6 & 0.1091 \\
7 & 0.0546 \\
8 & 0.0221 \\
9 & 0.0074 \\
10 & 0.0020 \\
11 & 0.0005 \\
12 & 0.0001 \\
$13 . .20$ & 0.0000
\end{tabular}

admit how many classes I took or taught before anyone mentioned the name Wald. In this era of computer software for statistical analysis, exact methods are more readily available than ever. Exact methods are necessary with small samples and sometimes necessary with large samples too. Approximations do not always work well even for large samples; if the rate of occurrence is very low (like a typical incidence density rate for central line infections), then $n p$ or $n q$ still may be smaller than required despite large values for $n$.

\section{REFERENCES}

1. Birnbaum D. Who is at risk of what? Infect Control Hosp Epidemiol 1999;20:706-707.

. Rothman KJ, Greenland S. Modern Epidemiology. 2nd ed. Philadelphia, PA: Lippincott-Raven Publishers; 1998.

\title{
Clinicians' Interpretation of PEP for Occupational Exposure to HIV
}

\section{Gina Pugliese, RN, MS Martin S. Favero, PhD}

Drs. David Bansberg and Ronald Goldschmidt from San Francisco General Hospital recently reported their experiences with the National Clinicians' Post-Exposure Prophylaxis Hotline (PEPline). The PEPline (1-888-HIV-4911), funded by the Health Resources and Services Administration and the $\mathrm{CDC}$, provides 24-hour-a-day advice regarding the management of occupational exposures to bloodborne pathogens for healthcare workers and the clinicians caring for them.

The authors provided 4,253 consultations in the first year of PEPline operation (November 1, 1997-October $31,1998)$ and recommended stopping or not starting PEP in the majority (58\%) of consultations. Seven percent of callers reported having initiated or receiving two- or three-drug regimens after an event that was not a true exposure. A common scenario was a request for advice about how to manage adverse drug effects that occurred after several days of a threedrug regimen that was started after an exposure to nonbloody saliva from an HIV-infected patient. Expert consultation and reassurance that the event is not a true exposure to bloodborne pathogens not only provide reassurance to the healthcare worker, but also prevents PEP-related adverse and toxic effects.

Expert interpretation of the guidelines also can be helpful in assisting in the choice of a regimen after an exposure from a patient who has taken several antiretroviral drugs. They point out the importance of choosing a regimen that maximizes biological activity against a virus previously exposed to several antiretroviral medications. However, given the rapid changes in our understanding of antiretroviral resistance, choosing an optimal regimen often extends beyond the scope of current guidelines and the knowledge base of many clinicians. Moreover, occupational exposures (or potential exposures) to HIV require urgent evaluation to provide appropriate counseling for an emotionally traumatic event, assess the risk of the exposure, explain the risks and benefits of antiretroviral prophylaxis, choose the optimal regimen, and manage adverse effects and toxicities during prophylaxis. The PEPline can assist in these simultaneous activities during the interpretation and application of current guidelines.

FROM: Bangsberg D, Goldschmidt RH. Postexposure prophylaxis for occupational exposure to HIV. JAMA 1999;282:1623-1624. 